\title{
The relationship of fear of movement with pain, range of motion and function in patients with shoulder pathologies
}

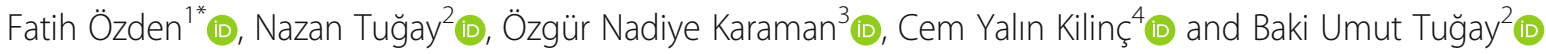

\begin{abstract}
Background: The pain, fear of movement, and disability are interrelated parameters that may adversely affect the individual in terms of medical and social aspects. This relationship has not been well studied in shoulder pathologies. We carried out a single-center cross-sectional study in the Orthopaedics and Traumatology Clinic. The aim of the study was to investigate the relationship between pain, function range of motion (ROM), and fear of movement in patients with shoulder pain.

Results: The mean age of the participants was $53.7 \pm 11.2$ years. Symptom duration was $11.65 \pm 23.39$ months. Most of the subjects (66.7\%) had impingement syndrome. There was a weak to moderate negative correlation between both visual analog scale (VAS) and Oxford Shoulder Score (OSS) with flexion, abduction, external rotation ROM values $\left(r_{1}=-0.293, r_{2}=-0.348, r_{3}=-0.330, p<0.001\right)$. In addition, there was a weak negative correlation between Tampa Kinesiophobia Score (TKS) and ROM values of abduction, internal rotation, and external rotation $\left(r_{1}\right.$ $\left.=-0.273, r_{2}=-0.207, r_{3}=-0.250, p<0.05\right)$. Pain was weakly and positively correlated with OSS $(r=0.209, p<$ 0.05).
\end{abstract}

Conclusions: According to our results, shoulder pain was associated with functionality, but fear of movement was not associated with shoulder pain or functionality. ROM was associated with both pain, functionality, and fear of movement.

Keywords: Shoulder, Kinesiophobia, Clinical assessment, Functionality

\section{Background}

Shoulder problems are the third most common condition in the musculoskeletal complaints [1-3]. It causes morbidity and disability which limits the individual's daily life, work, and social activities [4]. According to the data obtained from the general population, the prevalence of shoulder pain is $7-26 \%$, while the 1-year prevalence is $7-47 \%$ [5-7]. Due to the differences in the definition of shoulder problems in the literature, it is seen that this ratio varies and has a broad spectrum [2].

\footnotetext{
* Correspondence: fatihozden@mu.edu.tr

'Köyceğiz Vocational School of Health Services, Elderly Care Department, Muğla Sıtkı Koçman University, Köyceğiz, Muğla, Turkey

Full list of author information is available at the end of the article
}

According to the data obtained from cross-sectional and descriptive clinical studies, shoulder pain and loss of function have been shown to be related to each other $[5,8]$. Also, in particular, shoulder flexion and abduction were associated with shoulder pain and disability $[5,9,10]$. Recent studies have suggested that shoulder pain is affected by psychosocial and environmental factors and this leads to catastrophizing of pain $[5,11,12]$. Besides, according to a current systematic review, there is a relationship between pain, loss of function, and fear of movement [13].

"Pain-related fear" or "kinesiophobia" is defined as a psychophysiological, behavioral, and cognitive response that results from a painful stimulus [14]. In order to explain the fear of movement in detail and to explain its relationship with pain and disability based on evidence,

\section{Springer Open}

(c) The Author(s). 2021 Open Access This article is licensed under a Creative Commons Attribution 4.0 International License, which permits use, sharing, adaptation, distribution and reproduction in any medium or format, as long as you give appropriate credit to the original author(s) and the source, provide a link to the Creative Commons licence, and indicate if changes were made. The images or other third party material in this article are included in the article's Creative Commons licence, unless indicated otherwise in a credit line to the material. If material is not included in the article's Creative Commons licence and your intended use is not permitted by statutory regulation or exceeds the permitted use, you will need to obtain permission directly from the copyright holder. To view a copy of this licence, visit http://creativecommons.org/licenses/by/4.0/. 
it would be appropriate to mention the "Fear Avoidance Model (FAM)" developed by Lethem and colleagues in $1983[15,16]$. In this model, psychological factors such as fear of movement and pain catastrophizing have been shown to cause chronic pain and loss of function. Factors such as fear of movement and fear of pain caused by movement create a vicious circle with increasing pain and disability. For this reason, pain, fear of movement, and disability are interrelated parameters. This situation causes problems that may adversely affect the individual in terms of medical and social aspects [16].

Lentz et al. have proved that variables such as pain, disability, range of motion, duration of symptoms, and age provide important prognostic information about shoulder-related pathology [5]. There was no other study in the literature investigating the pain, function, fear of movement, and range of motion of the patients with shoulder problems, comparatively. Fear of movement could constrain the individual from using the range of motion efficiently and functionally during daily life activities. This situation may increase the limitations of movement and possible pain during movement maneuvers. Pain during movement can also trigger more kinesiophobia, thus creating a vicious circle between these three parameters. Subjective analysis of the relationship between kinesiophobia with other clinical parameters in the shoulder joint can reveal this vicious circle. Clinical important data will be obtained by observing the relationship between clinical parameters of an individual with a pathologic diagnosis of the musculoskeletal system in the shoulder joint. Clinical important data will be obtained by observing the relationship between clinical parameters of an individual with a pathologic diagnosis of the musculoskeletal system in the shoulder joint. Accordingly, it will be possible to determine the cycle of fear of movement in the monitoring of symptoms in clinical practice. The aim of the study was to investigate the relationship between pain, function range of motion (ROM), and fear of movement in patients with shoulder pain.

\section{Methods}

\section{Study design and participants}

We conducted a single-center cross-sectional study in the Orthopedics and Traumatology Clinic of Muğla Sttkı Koçman University Training and Research Hospital from 2017 to 2019. Initially, the orthopaedics and traumatology surgeon made the diagnosis and a total of $166 \mathrm{pa}-$ tients with shoulder pathologies were assessed for eligibility by the same surgeon. Sixty-four cases who did not meet the inclusion criteria were eliminated from the study. The inclusion criteria of the study were to be diagnosed as a musculoskeletal pathology of the shoulder joint and to be literate in Turkish. The exclusion criteria of the study were patients with shoulder joint surgery, shoulder fracture, glenohumeral dislocation, cancer, systemic diseases, cervicogenic symptoms, peripheral and central nervous system disorders, and refusal to participate in treatment. The study protocol was approved by the ethics committee of Muğla Sitkı Koçman University. All of the assessments were conducted in accordance with the Helsinki declaration. All cases provided a written informed consent approved by the ethics committee.

\section{Data collection}

After the diagnosis of shoulder pathology, the patients were evaluated immediately and were not subjected to physiotherapy or other conservative/surgical treatment. Data collection and all other evaluations were performed by a physiotherapist with 20 years of professional experience. Socio-demographic information of the cases were recorded. The pain at the activity was evaluated by visual analog scale (VAS). The shoulder active (ROM) was measured for shoulder flexion, abduction, internal, and external rotation (while shoulder was at $90^{\circ}$ abduction) with a universal goniometer using standard procedures [17].

The Turkish version of Oxford Shoulder Score (OSS) was used for functional status assessment and the Turkish version of Tampa Kinesiophobia Scale (TKS) was used for the assessment of fear of movement $[18,19]$. Study forms were filled, and questions were self-filled by the patients according to the guidelines. OSS is an assessment tool that examines shoulder joint function. Points between 12 and 20 indicates shoulder joint function is normal, $21-30$ mild to moderate shoulder arthritis, 31-40 medium-severe shoulder arthritis, and 4160 severe shoulder arthritis [20]. We used OSS due to its comprehensibility, ease of completion, short completion time, and its short structure [21]. TKS is a checklist of 17 questions and is used in acute and chronic low back pain, shoulder problems, fibromyalgia, and musculoskeletal injuries and whiplash-related diseases. The scale contains a 4-point Likert score $(1=$ Strongly disagree to $4=$ Strongly agree). A total score is calculated after the reversal of items $4,8,12$, and 16 . The total score ranged from 17 to 68 . The higher the score of the individual indicates a higher the kinesiophobia. Using the total score is preferred and recommended in the studies [14, 22].

\section{Statistical analysis}

The statistical package for the social sciences for Windows version 25 (SPSS Inc., Chicago, IL) computer program was used for data analysis. Quantitative variables were presented as mean \pm standard deviation $(\mathrm{X} \pm \mathrm{SD})$, range (minimum-maximum), and qualitative variables were presented as percent (\%). The Spearman rank correlation coefficient was used to investigate the 
relationship between the pain, ROM, disability, and fear of movement. The correlation coefficients were interpreted as $0-0.1=$ very low, $0.10-0.30=$ low, $0.30-0.50=$ moderate, $0.50-0.70=$ high, $0.70-0.90=$ very high, and $0.90-1.0=$ strong [23].

\section{Results}

A total of 102 patients (70 women (68.6\%), 32 men $(31.4 \%)$ ) with a mean age of $53.7 \pm 11.2$ years (range, $22-$ 79 years) were included in the study. Demographic information and characteristics of the patients are given in Table $1.68 .6 \%$ of the patients admitted to the clinic with shoulder problems were women.

The patients who presented to the outpatient clinic with the complaint of shoulder problem were diagnosed with subacromial impingement syndrome, rotator cuff ruptures, biceps tendonitis, and adhesive capsulitis. A vast majority $(66.7 \%)$ of the participants were diagnosed with subacromial impingement syndrome. Also, the symptom duration of shoulder problems in most of the subjects (69.6\%) persisted for 3 months or more. Clinical data are given in Table 2. The values of active range of motion and the VAS, OSS, and TKS scale scores of the patients are presented in Table 3.

The relationship between the ROM with VAS, OSS, and TKS are given in Table 4 and correlation between VAS, OSS, and TKS are given in Table 5. There was a weak to moderate negative correlation between both visual analog scale (VAS) and Oxford Shoulder Score (OSS) with flexion, abduction, external rotation range of motion $(\mathrm{ROM})$ values $\left(r_{1}=-0.293, r_{2}=-0.348, r_{3}=-\right.$ $0.330, p<0.001)$. In addition, there was a weak negative correlation between Tampa Kinesiophobia Score (TKS) and ROM values of abduction, internal rotation, and external rotation $\left(r_{1}=-0.273, r_{2}=-0.207, r_{3}=-0.250\right.$, $p<0.05)$. According to the results of the correlation

Table 1 Socio-demographic information and characteristics of the patients

\begin{tabular}{ll}
\hline & Total $(\boldsymbol{n}=\mathbf{1 0 2})$ \\
\hline Age (years, mean \pm SD) & $53.7 \pm 11.2$ \\
Height $(\mathbf{m}$, mean \pm SD) & $1.65 \pm 0.08$ \\
Weight $(\mathbf{k g}$, mean \pm SD) & $76.00 \pm 12.94$ \\
BMI $\left(\mathbf{k g} / \mathbf{m}^{2}\right.$, mean \pm SD) & $27.74 \pm 4.01$ \\
Education & \\
Primary school $(n, \%)$ & $60(58.9)$ \\
High school $(n, \%)$ & $17(16.6)$ \\
Post-secondary $(n, \%)$ & $25(24.5)$ \\
Residence & \\
Urban $(n, \%)$ & $78(76.5)$ \\
Rural $(n, \%)$ & $24(23.5)$ \\
\hline
\end{tabular}

$S D$ standard deviation, $n$ number of patients, BMI body mass index
Table 2 Clinical data of the patients

\begin{tabular}{ll}
\hline & Total $(\boldsymbol{n}=\mathbf{1 0 2})$ \\
\hline Affected shoulder & $61(59.8)$ \\
Right $(n, \%)$ & $41(40.2)$ \\
Left $(n, \%)$ & \\
Diagnosis & $68(66.7)$ \\
$\quad$ Subacromial impingement syndrome $(n, \%)$ & $20(19.6)$ \\
$\quad$ Rotator cuff ruptures $(n, \%)$ & $6(5.9)$ \\
Bicep tendinitis $(n, \%)$ & $8(7.8)$ \\
Adhesive capsulitis $(n, \%)$ & \\
Chronic disease & $55(53.9)$ \\
$\quad$ Yes ( $n, \%)$ & $47(46.1)$ \\
No $(n, \%)$ & $11.65 \pm 23.39$ \\
Symptom duration (months)
\end{tabular}

$S D$ standard deviation, $n$ number of patients

analysis for the subjective evaluation parameters reported by the patient, only OSS and VAS were found to be weakly and positively correlated $(r=0.209, p<0.05)$ (Table 5). There was no correlation between TKS with OSS and VAS $\left(r_{1}=0.079, r_{1}=0.027, p>0.05\right)$.

\section{Discussion}

The present study showed that shoulder pain was associated with functionality. However, fear of movement was not associated with neither shoulder pain nor function. An increase in pain level leads to a decrease in functionality. As a matter of fact, in many studies, it has been shown that pain level and functionality are related in different patient groups with shoulder problems [5, 10, 24, 25].

ROM was associated with both pain, functionality, and fear of movement. Over a long period of time, hypervigilance, and avoidance of movement cause to immobilization complications such as deconditioning which provokes more pain and functional impairment [26]. There was a weak to moderate correlation between ROM (flexion, abduction, external rotation) and VAS.

Table 3 Average values (standard deviation, min-max) for ROM, VAS, OSS and TKS

\begin{tabular}{lll}
\hline $\mathbf{n : 1 0 2}$ & Mean \pm SD & Range \\
\hline ROM $\left(^{\circ}\right)$ & $145.98 \pm 32.23$ & $(60-180)$ \\
$\quad$ Flexion & $128.77 \pm 42.78$ & $(30-180)$ \\
Abduction & $60.44 \pm 15.48$ & $(0-75)$ \\
Internal rotation & $63.87 \pm 27.61$ & $(0-90)$ \\
$\quad$ External rotation & $6.81 \pm 2.33$ & $(1.3-10)$ \\
VAS & $31.26 \pm 12.77$ & $(6.59)$ \\
OSS & $40.63 \pm 5.67$ & $(23-56)$ \\
TKS &
\end{tabular}

$S D$ standard deviation, $n$ number of patients, VAS visual analog scale, $R O M$ range of motion, OSS Oxford Shoulder Score, TKS Tampa Kinesiophobia Scale 
Table 4 Correlation between ROM with VAS, OSS, and TKS

\begin{tabular}{llll}
\hline $\boldsymbol{n}=102$ & VAS & OSS & TKS \\
\hline ROM & & & \\
Flexion & $-0.293^{* *}$ & $-0.295^{* *}$ & -0.193 \\
Abduction & $-0.348^{* *}$ & $-0.311^{* *}$ & $-0.273^{* *}$ \\
Internal rotation & -0.169 & 0.016 & $-0.207^{*}$ \\
External rotation & $-330^{* *}$ & $-0.336^{* *}$ & $-0.250^{*}$
\end{tabular}

VAS visual analog scale, ROM range of motion, OSS Oxford Shoulder Score, TKS Tampa Kinesiophobia Scale

${ }^{*} p<0.05,{ }^{* *} p<0.001$

As the pain level increased, these ROM values decreased. However, no relationship was observed with internal rotation. It has been reported that individuals with subacromial impingement syndrome generally have an increase in internal rotation compared to healthy cases. This may be due to this phenomenon. A similar condition was also observed between OSS and ROM. There was a weak to moderate correlation between OSS and all ROM values except internal rotation. Fear of movement was not associated only with flexion. There was a relationship for the other three $\mathrm{ROM}$ parameters. In the study of Lentz et al., there was no correlation between ROM (flexion, abduction, internal rotation, external rotation) and fear of movement. In addition, only the flexion ROM was found to be correlated with TKS-11 [5]. In the study performed by Anwer et al. ROM (flexion, extension, abduction, internal rotation, external rotation) was found to be associated with both pain and functionality [10]. When the literature is examined, it is seen that there is a small number of studies in which shoulder joint ROM and kinesophobia are examined. There should be more studies in order to examine these parameters and to compare the results obtained.

TKS was not correlated with either VAS or OSS. In other words, the fear of shoulder movement is not related to the pain level and functionality of these cases. In a cross-sectional study, kinesiophobia was not associated with pain but was associated with functionality [5]. In another study conducted in 2014, clinical variables (pain, functionality) were found to be associated with fear avoidance [8].

In a systematic review with a higher level of evidence by Martinez-Calderon and colleagues' pain and

Table 5 Correlation between VAS, OSS and TKS

\begin{tabular}{ll}
\hline $\boldsymbol{n}=102$ & $\boldsymbol{r}$ \\
\hline VAS-TKS & 0.027 \\
OSS-VAS & $0.209^{*}$ \\
TKS-OSS & 0.079
\end{tabular}

$r$ Spearman rank correlation coefficient, VAS visual analog scale, OSS Oxford Shoulder Score, TKS Tampa Kinesiophobia Scale

${ }^{*} p<0.05$ functionality were shown to be associated with psychological pain beliefs of the individual (e.g., kinesiophobia, pain catastrophising) [13]. However, some studies in this review also show that kinesiophobia is not associated with pain and disability. There are some limitations of the study. Kinesophobia may not be associated with pain and disability due to reasons such as the fact that the sample size of our study did not consist of a homogenous group with the same pathology, individuals fear of movement was observed at different levels in acute, subacute, chronic or severe cases, or that the sample size was insufficient for this cross-sectional study. Correlations between clinical parameters are moderate to low. To be able to reveal the relationship between the two parameters more clearly, the stronger the correlation coefficient would be essential in order to increase the evidence level of the study. However, these preliminary results of our study, which has been carried out with a pragmatic approach, may contribute to the formation of the hypotheses of more comprehensive cohort or case-control studies. Conducting the study with higher sample size and more comprehensive methods in which the cases are selected by grouping with more accurate inclusion/exclusion criteria and included in different psychological parameters will provide higher level of evidence.

\section{Conclusion}

According to our results, shoulder pain was associated with functionality. Fear of movement was not associated with shoulder pain or functionality. ROM was associated with both pain, functionality and fear of movement. It may be concluded that in patients with shoulder problems, the symptoms are more likely to be confronted with fear of movement when they reach a condition that will limit joint movements.

\section{Abbreviations}

FAM: Fear Avoidance Model; VAS: Visual analog scale; ROM: Range of motion; OSS: Oxford Shoulder Score; TKS: Total knee arthroplasty; SD: Standard deviation; $n$ : Number of patients; $r$ : Spearman rank correlation coefficient

\section{Acknowledgements}

Not applicable.

\section{Authors' contributions}

FÖ and NT researched literature and conceived the study. FÖ, NT, ÖNK, BUT, and CYK were involved in protocol development, gaining ethical approval, patient recruitment, and data analysis. FÖ and NT wrote the first draft of the manuscript. The authors reviewed and edited the manuscript and approved the final version of the manuscript.

\section{Funding}

Not applicable.

Availability of data and materials Not applicable. 


\section{Ethics approval and consent to participate}

The study protocol was approved by the ethics committee of Muğla University (17-12-2014). All of the assessments were conducted in accordance with the Helsinki declaration. All cases provided a written informed consent approved by the ethics committee.

\section{Consent for publication}

Not applicable.

\section{Competing interests}

The authors declare that they have no competing interests.

\section{Author details}

${ }^{1}$ Köyceğiz Vocational School of Health Services, Elderly Care Department, Muğla Sıtkı Koçman University, Köyceğiz, Muğla, Turkey. ${ }^{2}$ Faculty of Health Sciences, Department of Physiotherapy and Rehabilitation, Muğla Sıtk Koçman University, Menteşe, Muğla, Turkey. ${ }^{3}$ Training and Research Hospital, Physical Therapy and Rehabilitation Unit, Muğla Sıtkı Koçman University, Menteşe, Muğla, Turkey. ${ }^{4}$ Faculty of Medicine, Department of Orthopedics and Traumatology, Muğla Sıtkı Koçman University, Menteşe, Muğla, Turkey.

Received: 21 October 2020 Accepted: 26 January 2021

Published online: 24 February 2021

\section{References}

1. Wolfensberger A, Vuistiner $\mathrm{P}$, Konzelmann M, Plomb-Holmes C, Léger B, Luthi F. Clinician and patient-reported outcomes are associated with psychological factors in patients with chronic shoulder pain. Clin Orthop Relat Res. 2016:474(9):2030-9.

2. Luime JJ, Koes BW, Hendriksen IJM, Burdorf A, Verhagen AP, Miedema HS, et al. Prevalence and incidence of shoulder pain in the general population; a systematic review. Scand J Rheumatol. 2004;33(2):73-81.

3. Greving K, Dorrestijn O, Winters JC, Groenhof F, Van der Meer K, Stevens M, et al. Incidence, prevalence, and consultation rates of shoulder complaints in general practice. Scand J Rheumatol. 2012;41(2):150-5.

4. Linsell L, Dawson J, Zondervan K, Rose P, Randall T, Fitzpatrick R, et al. Prevalence and incidence of adults consulting for shoulder conditions in UK primary care; patterns of diagnosis and referral. Rheumatology. 2005;45(2): 215-21.

5. Lentz TA, Barabas JA, Day T, Bishop MD, George SZ. The relationship of pain intensity, physical impairment, and pain-related fear to function in patients with shoulder pathology. J Orthop Sports Phys Ther. 2009;39(4):270-7.

6. Nygren A, Berglund A, Von Koch M. Neck-and-shoulder pain, an increasing problem. Strategies for using insurance material to follow trends. Scand J Rehabil Med Suppl. 1995;32:107

7. Tekavec E, Jöud A, Rittner R, Mikoczy Z, Nordander C, Petersson IF, et al. Population-based consultation patterns in patients with shoulder pain diagnoses. BMC Musculoskelet Disord. 2012;13(1):238.

8. Kromer TO, Sieben JM, de Bie RA, Bastiaenen CHG. Influence of fearavoidance beliefs on disability in patients with subacromial shoulder pain in primary care: a secondary analysis. Phys Ther. 2014;94(12):1775-84.

9. Fayad F, Roby-Brami A, Gautheron V, Lefevre-Colau M-M, Hanneton S, Fermanian J, et al. Relationship of glenohumeral elevation and 3dimensional scapular kinematics with disability in patients with shoulder disorders. J Rehabil Med. 2008:40(6):456-60.

10. Anwer $\mathrm{S}$, Alghadir AH, Al-Eisa ES, lqbal ZA. The relationships between shoulder pain, range of motion, and disability in patients with shoulder dysfunction. J Back Musculoskel Rehabil. 2018;31(1):163-7.

11. George SZ, Hirsh AT. Psychologic influence on experimental pain sensitivity and clinical pain intensity for patients with shoulder pain. J Pain. 2009;10(3): 293-9.

12. Roe Y, Soberg HL, Bautz-Holter E, Ostensjo S. A systematic review of measures of shoulder pain and functioning using the International classification of functioning, disability and health (ICF). BMC Musculoskelet Disord. 2013;14(1):73

13. Martinez-Calderon J, Struyf F, Meeus M, Luque-Suarez A. The association between pain beliefs and pain intensity and/or disability in people with shoulder pain: a systematic review. Musculoskelet Sci Pract. 2018;37:29-57.

14. Mintken PE, Cleland JA, Whitman JM, George SZ. Psychometric properties of the Fear-Avoidance Beliefs Questionnaire and Tampa Scale of Kinesiophobia in patients with shoulder pain. Arch Phys Med Rehabil. 2010;91(7):1128-36.
15. Lethem J, Slade PD, Troup JDG, Bentley G. Outline of a fear-avoidance model of exaggerated pain perception-I. Behav Res Ther. 1983:21(4):401-8.

16. Leeuw M, Goossens MEJB, Linton SJ, Crombez G, Boersma K, Vlaeyen JWS. The fear-avoidance model of musculoskeletal pain: current state of scientific evidence. J Behav Med. 2007;30(1):77-94.

17. Norkin CC, White DJ. Measurement of joint motion: a guide to goniometry. Philadelphia: FA Davis; 2003

18. Yilmaz ÖT, Yakut Y, Uygur F, Uluğ N. Tampa Kinezyofobi Ölçeği'nin Türkçe versiyonu ve test-tekrar test güvenirliği. Fiz Rehabil. 2011;22(1):44-9.

19. Tuğay U, Tuğay N, Gelecek N, Özkan M. Oxford Shoulder Score: crosscultural adaptation and validation of the Turkish version. Arch Orthop Trauma Surg. 2011;131(5):687-94

20. Dawson J, Fitzpatrick R, Carr A, Murray D. Questionnaire on the perceptions of patients about total hip replacement. J Bone Joint Surg Br. 1996;78(2):185-90.

21. Angst F, Schwyzer HK, Aeschlimann A, Simmen BR, Goldhahn J. Measures of adult shoulder function: disabilities of the arm, shoulder, and hand questionnaire (DASH) and its short version (QuickDASH), shoulder pain and disability index (SPADI), American shoulder and elbow surgeons (ASES) society standardized shoulder assessment form, constant (Murley) score (CS), simple shoulder test (SST), oxford shoulder score (OSS), shoulder disability questionnaire (SDQ), and Western Ontario shoulder instability index (WOSI). Arthritis Care Res. 2011:63(S11):S174-S88.

22. Vlaeyen JWS, Kole-Snijders AMJ, Boeren RGB, Van Eek H. Fear of movement/ (re) injury in chronic low back pain and its relation to behavioral performance. Pain. 1995;62(3):363-72.

23. Reis MM, Oliveira FG, Lopes ÉM. Analysis of reference evapotranspiration of Janaúba, mg. Idesia (Chile). 2016;34(4):1-8.

24. Mitchell C, Adebajo A, Hay E, Carr A. Shoulder pain: diagnosis and management in primary care. BMJ. 2005;331(7525):1124-8.

25. Unruh KP, Kuhn JE, Sanders R, An Q, Baumgarten KM, Bishop JY, et al. The duration of symptoms does not correlate with rotator cuff tear severity or other patientrelated features: a cross-sectional study of patients with atraumatic, full-thickness rotator cuff tears. J Shoulder Elb Surg. 2014:23(7):1052-8.

26. Vlaeyen JWS, Linton SJ. Fear-avoidance and its consequences in chronic musculoskeletal pain: a state of the art. Pain. 2000:85(3):317-32.

\section{Publisher's Note}

Springer Nature remains neutral with regard to jurisdictional claims in published maps and institutional affiliations.

\section{Submit your manuscript to a SpringerOpen ${ }^{\circ}$ journal and benefit from:}

- Convenient online submission

- Rigorous peer review

- Open access: articles freely available online

- High visibility within the field

Retaining the copyright to your article

Submit your next manuscript at $>$ springeropen.com 